\title{
Improving induced abortion care in Scotland: enablers and constraints
}

\author{
Lale Say, Robbie Foy
}

\begin{abstract}
Background Induced abortion is the most common gynaecological procedure in Scotland. Despite several recent initiatives to improve the quality of abortion care, inappropriate variations in care remain
\end{abstract}

Objective To identify and explore factors that enable or constrain the provision of high-quality induced abortion care in Scotland.

Methods Interviews with a range of key informants with differing perspectives and levels of involvement in abortion care. The interview framework identified factors related to recommendations, targeted individuals, or the organisation and wider environment that enable or inhibit evidence-based practice.

Results Induced abortion care in Scotland is generally perceived to be of good quality but the need for further action to tackle important inappropriate variations in care is recognised. Some aspects of care can be improved by tackling individual-level barriers and providing better evidence to support change. Some individual-level barriers (e.g. attitudes) are less amenable to change than others (e.g. knowledge). However, major barriers to quality improvement are rooted in organisational and socia culture.

Conclusion Tackling variations in abortion care requires a multilevel approach targeting both individual factors and organisational culture.

J Fam Plann Reprod Health Care 2005; 31(1): 20-23 (Accepted 4 June 2004)

\section{Key message points}

- In general terms, abortion care in Scotland is perceived to be of good quality, but inappropriate variations in care exist.

- Some aspects of care can be improved by tackling individual-level barriers and providing better evidence to support change, but problems are rooted in organisational and social culture

- Tackling variations in abortion care requires a multilevel approach targeting both individual factors and organisational culture.

Department of Reproductive Health and Research, World Health Organization, Geneva, Switzerland Lale Say, MD, MSc, Scientist

Centre for Health Services Research, University of Newcastle-upon-Tyne, Newcastle-upon-Tyne, UK Robbie Foy, MFPHM, MRCGP, Clinical Senior Lecturer in Primary Care

Correspondence to: Dr Lale Say, Department of Reproductive Health and Research, World Health Organization, 20 Avenue Appia, CH-1211 Geneva 27, Switzerland. Tel: +4122 7914816. Fax: +4122 791 4171. E-mail: sayl@who.int

\section{Introduction}

Induced abortion is the most common gynaecological procedure in Scotland, comprising $12 \%$ of gynaecological inpatient and day cases. ${ }^{1}$ Over $99 \%$ of induced abortions took place in National Health Service (NHS) hospitals in Scotland in 2000.2 Several initiatives to improve the quality of abortion care have taken place there over the last decade, including a professionally-led standard setting and audit project and the dissemination of an evidence-based guideline by the UK Royal College of Obstetricians and Gynaecologists (RCOG). ${ }^{3,4}$ The guideline promoted equitable access to services across the UK, the use of more effective interventions, and improved communication with women. Most recently, all Scottish gynaecology units participated in the Improving Abortion Care Trial (ImpACT). 5 This found no effect of a multifaceted strategy, including clinical audit and educational meetings, to support implementation of the RCOG guideline. ImpACT findings suggested that abortion care had improved over the preceding decade but that inappropriate variations in care remained, especially in relation to access and aftercare. However, the strategy was unable to overcome wider organisational and environmental barriers outside the control of professionals providing abortion care. We interviewed a range of key informants to further identify and explore factors that enable or constrain the provision of high-quality induced abortion care in Scotland.

\section{Methods}

Framework

A range of frameworks have been used to describe factors that may enable or inhibit evidence-based practice..$^{6-11} \mathrm{We}$ used a pragmatic approach that describes factors under three main categories related to the nature of the evidence or change itself, the characteristics of the individuals who need to change, and the characteristics of the organisation or wider environment (Box 1).12

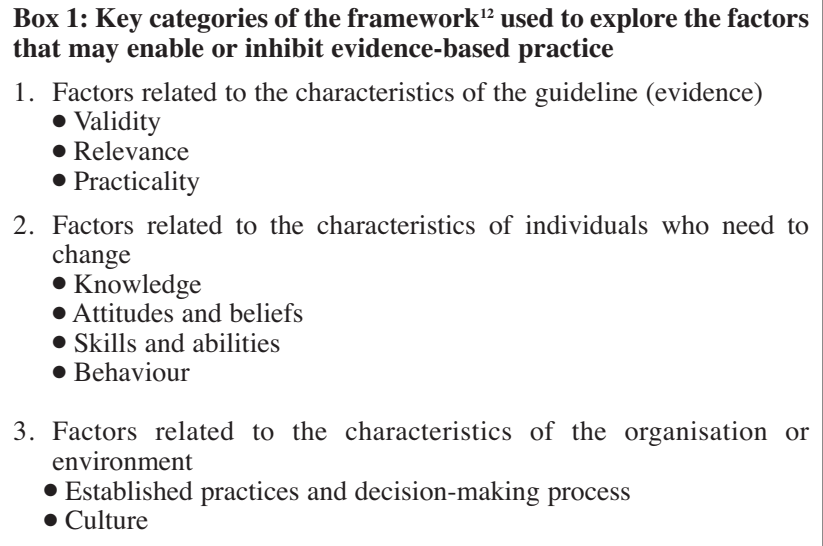

\section{Interview schedule}

We developed a semi-structured interview schedule covering the framework headings to identify factors that enabled or constrained the development of induced abortion care. Other topics explored included views on the general quality of care and what aspects of provision required further action. 


\section{Informants}

We purposively selected eight informants with varying experiences of service involvement so as to represent a range of perspectives of abortion care in Scotland (Box 2).

\section{Box 2: Range of key informants interviewed}

- A consultant/director of family planning and well woman services in an NHS Primary Care Trust

- The programme co-ordinator of a national clinical effectiveness programme

- A consultant in public health medicine (responsibility for women and children)

- A (male) consultant gynaecologist with an interest in abortion care - A (female) consultant gynaecologist with limited involvement in
abortion care

- A nursing sister working in abortion services

- A general practitioner

- A researcher from the Scottish Association of Local Health Councils (which then represented patient interests within the NHS in Scotland)

\section{Procedure and analysis}

The interview schedule was pre-tested and revised prior to use. One of the authors (L.S.) conducted face-to-face interviews, which lasted around $45 \mathrm{~min}$ each. All questions included in the schedule were covered, with the order of questions partly being determined by responses. Interviews were not tape-recorded but notes were taken and written up immediately afterwards. We analysed the data by grouping common themes according to the pre-existing categories of the aforementioned framework. ${ }^{12}$ In order to preserve confidentiality, we linked each quotation to informants by numbers only. The interviews were conducted in mid-2001, coincident with the ImpACT intervention period.

\section{Results}

The need for high-quality care

It was generally acknowledged that high-quality care should address women's needs and concerns at a time when they are vulnerable (Box 3). Most informants did recognise that major improvements in abortion care had taken place within Scotland over a number of years, albeit at varying rates across different regions. Yet several respondents pointed out that abortion services were relatively neglected and 'needed advocacy'. Current variations in the quality of care among gynaecology units were widely accepted. Quality also varied within units; whilst certain technical aspects of care were generally well delivered (e.g. safety, screening for infections), other aspects were paid less attention (e.g. aftercare).

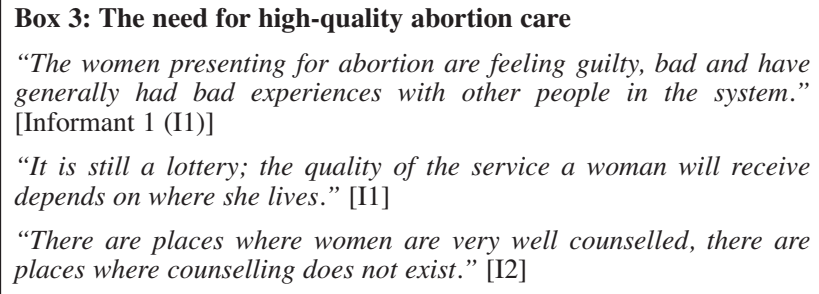

"There are places where women are very well counselled, there are places where counselling does not exist." [I2]

\section{Guideline-related factors}

An evidence-based summary was welcomed; as well as addressing issues around effectiveness and efficiency, information on safety and risks were particularly important to women (Box 4). Expert-based guideline recommendations were more difficult to accept than those based upon more rigorous evidence. For example, one
RCOG guideline recommendation, namely that of offering a follow-up appointment within 2 weeks of the abortion procedure, was often not adhered to because of perceived insufficient evidence of benefit.

Box 4: Guideline-related factors
• Enablers
"[It is important] to relieve women's worries, for example, like the
assumption that abortion causes infertility. It is not the evidence." [I3]
"[It is important] to be sure that we are giving safe, effective and cost-
effective services." [I4]
- Restraints
"I think it [abortion] is very painful with local anaesthesia ... more
likely to get retained products." [I3]

\section{Individual factors}

On the whole, there was 'a level of commitment' and 'a lot of goodwill' among gynaecologists to improve care. However, the local development of abortion services often depended on key individuals (Box 5). Negative attitudes of gynaecologists towards abortion care had significantly restrained service development in some hospitals. Such attitudes were rooted in different perceptions. Most gynaecologists did not prioritise abortion care; it was not 'real gynaecology'. Because the procedure was common, it took time away from other 'important' gynaecological procedures.

Box 5: Individual factors

- Enablers

"Quality of care depends on a clinician caring about the service; if that individual does not exist, that hospital does it badly." [I8]

\section{- Restraints}

"Hospital-based gynaecologists see abortion patients reluctantly." [I5]

"They look at [abortion care] as a nuisance. Sometimes this is quite obstructive and acts as a barrier, not consciously, but by really being unhelpful." [I1]

"Most of the time [health professionals] are dealing to preserve life, helping women to achieve a good childbirth." [I3]

"Some of the GPs say dreadful things to women." [I1]

"[Gynaecologists] do not see developing the necessary skills for abortion as a priority." [I4]

"Doctors say nurses do not want to do medical abortions; they use nurses as an excuse not to set up a service." [I1]

Abortion also created perceived ethical conflicts (e.g. between preserving and ending life) and justification for religious and moral objections. Some gynaecologists believed the problem was rooted in women's own faults, and were intolerant and judgmental towards women requesting abortion. Male and junior doctors were seen as particularly intolerant. Negative attitudes to abortion also existed amongst general practitioners (GPs), which hindered the speed or quality of referrals.

There were arbitrary upper gestational age limits to perform abortions; some gynaecologists were performing abortions for gestations no later than 15-16 weeks, or even 12 weeks. Individual preferences, 'without logic', caused variable access to care.

Lack of knowledge and skills among gynaecologists were barriers to performing certain procedures. For example, the near-universal use of general anaesthesia for surgical abortions partly reflected clinicians' unfamiliarity with local anaesthesia. The introduction of local anaesthesia was further constrained by uncertainties over its benefits and acceptability to women. Many individuals naturally resisted change, even in the face of a clear 


\section{ARTICLE}

rationale or supporting evidence. The enhanced role of nurses could help expand services, but both the lack of selfconfidence among nurses and the lack of doctors' confidence in nurses limited action on this opportunity.

\section{Organisational and environmental factors}

The establishment of dedicated abortion clinics, multidisciplinary teams, and facilities (e.g. theatre lists) within an increasingly centralised system of abortion care represent key organisational developments. Informants believed that the establishment of an effective referral system had improved access..$^{13}$ In one city, directly linking family planning services to abortion clinics was considered to have improved access to appointments. Such developments were dependent upon an 'organisational commitment', in particular the prioritisation of dedicated resources by health boards (Box 6). For example, the expanded role of nursing staff in medical abortion was hindered by shortfalls in NHS training budgets to ensure education in the legal, technical and emotional aspects of abortion.

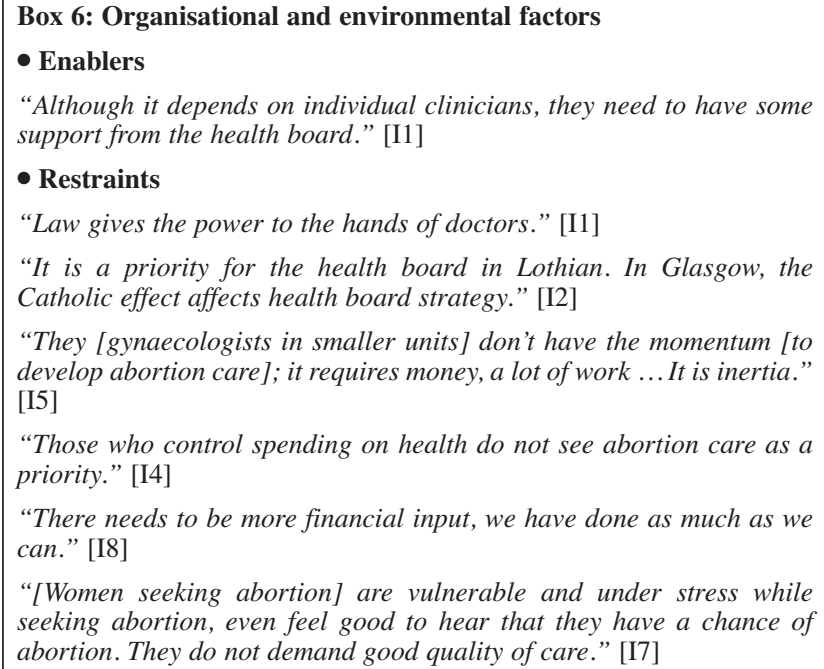

"Although it depends on individual clinicians, they need to have some support from the health board." [I1]

$\bullet$ Restraints

"Law gives the power to the hands of doctors." [I1]

"It is a priority for the health board in Lothian. In Glasgow, the Catholic effect affects health board strategy." [I2]

"They [gynaecologists in smaller units] don't have the momentum [to develop abortion care]; it requires money, a lot of work ... It is inertia." [I5]

"Those who control spending on health do not see abortion care as a priority." [14]

"There needs to be more financial input, we have done as much as we can." [I8]

"[Women seeking abortion] are vulnerable and under stress while seeking abortion, even feel good to hear that they have a chance of abortion. They do not demand good quality of care." [17]

Organisational commitment was shaped within the broader contexts of history, social networks and cultural expectations. The historical impact of influential individuals in Scotland who 'brought respect to abortion as a reproductive health care topic' was acknowledged. The continuing interest of a critical mass of Scottish gynaecologists in the development of medical abortion methods promoted its rapid introduction. The relatively small community of gynaecologists in Scotland also seemed to promote good care because it created a medium that allowed 'peer pressure' and greater influence. However, the close-knit nature of this community facilitated disproportionate negative as well as positive influences of key individuals. Such political influences partly explained the legacy of an East-West divide in the development of abortion services. The power of Roman Catholicism was also seen as a restraining factor that affected health board policy in the West. Cultural factors also influenced current legislation, ${ }^{14}$ labelled as 'repressive' by one informant because it strengthened the power of individual gynaecologists.

Women seeking abortion care had low expectations and differed from other gynaecology patients. They were generally from poorer social circumstances, younger and less articulate. These characteristics made it difficult for them to use the system properly, and to identify their choices and rights. They also felt guilty, shamed and stigmatised whilst seeking abortion care and were reluctant to criticise the service. Women's preferences sometimes might hinder adherence to guideline recommendations; some women, for instance, were reluctant to attend for follow-up so as to avoid seeing the same professionals who were involved in their abortion.

\section{Discussion}

In general terms, abortion care in Scotland is perceived to be of good quality - but further action is needed to address continuing inappropriate variations in care. This action partly involves tackling individual-level barriers and providing high-quality evidence to support change. Some of these barriers (e.g. attitudes) are less amenable to change than others (e.g. knowledge). However, the overriding message is that variations in care are rooted in organisational and social culture.

There are obvious limitations to this study. First, the sample of informants was small and biased towards individuals with an interest in improving abortion care. Nevertheless, the diversity of the sample enhanced identification of a broad range of factors that enabled or constrained the development of abortion care. Second, some distinctions between framework categories were relatively arbitrary: women's expectations could be viewed as individual or environmental influences. Third, the rigour of our methods - especially the interviews and analysis was constrained by our own limited resources. However, professionals in service settings who wish to identify barriers to change for other clinical problems may be similarly constrained to the use of expedient methods. Nevertheless, the factors identified by the interviews are consistent with those identified elsewhere, including from questionnaire surveys of gynaecologists, 15,16 a review of the organisation of services, 17 audit reports $1,18,19$ and an analysis of abortion trends in Scotland. ${ }^{20}$ Moreover, the use of our simple framework ensured the identification of wider organisational and cultural influences.

Major problems in abortion care exist, even within a well-developed system. Tackling these problems requires a multilevel approach. ${ }^{10}$ At one end of the spectrum, targeting individual professionals is unlikely by itself to reduce inappropriate variations in care. At the other, advocacy that targets both social attitudes and expectations and the legislative framework is also necessary but not sufficient by itself. Clinical governance occupies much of the ground between individual and environmental factors. It represents an attempt to change organisational culture and bring together a range of quality improvement methods to improve health care. ${ }^{21}$ Improving abortion care presents a distinct challenge for clinical governance.

Acknowledgement

The authors are grateful to the informants who participated in this study.

\section{Statements on funding and competing interests}

Funding. Lale Say received a Wellcome Trust Fellowship for the MSc Research Training Programme in Reproductive Health at the University of Edinburgh. Robbie Foy was funded by a joint Medical Research Council/Chief Scientist Office Special Training Fellowship in Health Services Research.

Competing interests. None identified.

References

1 Information and Statistics Division of the National Health Service in Scotland. SMR1 Returns for Gynaecological Operations 2002.
Edinburgh, UK: Information and Statistics Division, 2004.

2 Information and Statistics Division of the National Health Service in Scotland. Abortion Statistics Scotland 2002. http://www.isdscotland. org [Accessed 20 February 2004].

3 Penney GC, Glasier A, Templeton A. Agreeing criteria for audit of the management of induced abortion: an approach by national consensus survey. Qual Health Care 1993; 2: 167-169.

4 Royal College of Obstetricians and Gynaecologists (RCOG). The Care of Women Requesting Abortion. London, UK: RCOG, 2000. 
5 Foy R, Penney GC, Grimshaw JM, Ramsay CR, Walker AE, MacLennan $\mathrm{G}$, et al. A randomised controlled trial of a tailored multifaceted strategy to promote implementation of a clinical guideline on induced abortion care. Br J Obstet Gynaecol 2004; 111 726-733.

6 Davis DA, Taylor-Vaisey A. Translating guidelines into practice. A systematic review of theoretic concepts, practical experience and research evidence in the adoption of clinical practice guidelines. Can Med Assoc J 1997; 157: 408-416.

7 Mäkelä M, Thorsen T. A framework for guideline implementation studies. In: Thorsen T, Mäkelä M (eds), Changing Professional Practice. Theory and Practice of Clinical Guidelines Implementation. Copenhagen, Denmark: Danish Institute for Health Services Research and Development, 1999; 23-53.

8 Cabana MD, Rand CS, Powe NR, Wu AW, Wilson MH, Abboud PA, et al. Why don't physicians follow clinical practice guidelines? A framework for improvement. JAMA 1999; 282: 1458-1465.

9 Rubenstein LV, Mittman BS, Yano EM, Mulrow CD. From understanding health care provider behaviour to improving health care. The QUERI Framework for Quality Improvement. Med Care 2000; 38(Suppl.): I129-I141.

10 Ferlie EB, Shortell SM. Improving the quality of health care in the United Kingdom and the United States: a framework for change. Milbank $Q$ 2001; 79: 281-315.
11 Grol R. Beliefs and evidence in changing clinical practice. BMJ 1997 ; 315: $418-421$.

12 Foy R, Walker AE, Penney GC. Barriers to clinical guidelines: the need for concerted action. Br J Clin Governance 2001; 6: 166-174.

13 Glasier A, Thong JK. The establishment of a centralised referral service leads to earlier abortion. Health Bull (Edinb) 1991; 49: 254-259.

14 Abortion Act 1967. London, UK: Her Majesty's Stationery Office, 1967

15 Chamberlain A. Consultant gynaecologists and attitudes towards abortion. J Biosoc Sci 1980; 12: 407-415.

16 Francome C, Savage WD. Gynaecologists' abortion practice. $\mathrm{Br}$ Obstet Gynaecol 1992; 99: 153-157.

17 Glasier A. The organisation of abortion services. Curr Obstet Gynaecol 1993; 3: 23-27.

18 Penney GC, Templeton A. Impact of a national audit project on gynaecologists in Scotland. Qual Health Care 1994; 3: 1-3.

19 Penney GC, Templeton A, Glasier A. Patients' views on abortion care in Scottish Hospitals. Health Bull 1994; 52: 431-437.

20 Wilson MGA. Two decades of "legal" abortion in Scotland: a spatiotemporal analysis. Scott Geogr J 2000; 116: 1-23.

21 Scally G, Donaldson LJ. Clinical governance and the drive for quality improvement in the new NHS in England. BMJ 1998; 317: $61-65$.

\section{Book Reviews}

The Ovary (2nd edn). Peter C K Leung and Eli Y Adashi (eds). London, UK: Academic Press, 2004. ISBN: 012444562 4. Price: $£ 111.00$. Pages: 664 (hardback)

It is easy for those delivering health care at the 'pitface' to forget that virtually all clinical problems arising in reproductive health are controlled by the ovaries. Hidden from view within the abdominal cavity, the ovaries secrete hormones that produce profound changes in target organs such as the uterus, vagina, breast and bones. Modern reproductive health care involves manipulation of ovarian function, not only for contraception, but also for the management of a range of sex hormonedependent conditions such as menorrhagia, dysmenorrhoea, infertility and endometriosis. Some understanding of the physiological control of ovarian function is, therefore, a great help in the rational management of reproductive disorders.

This multi-author book is a very comprehensive account of the physiology of the ovary. The second edition has been extensively updated to include the most recent publications in a rapidly expanding area of science. The book covers hormonal and molecular mechanisms of follicular development, ovulation, oocyte maturation, and formation and function of the corpus luteum. Disappointingly, for the development of a once-a-month pill the mechanism underlying regression of the corpus luteum in our own species, in contrast to many other mammals, still remains a mystery despite extensive research.

Probably of more direct relevance to reproductive health professionals are chapters on induction of ovulation, infertility and assisted conception, and polycystic ovary disease. There is an excellent chapter by Joe Leigh Simpson and Aleksander Rajkovic on syndromes of ovarian failure that are associated with a number of gene mutations and molecular perturbations. For example, the fragile $\mathrm{X}$ syndrome of mental retardation and associated facial characteristics in males is caused by a mutation of the FMRI gene males is caused by a mutation of the FMRI gene
on Xq27, which results in repetition of triplet repeat (CGG) 230 times or more. In heterozygote females the clinical phenotype is related to the number of CGG repeats. Those women with 50-200 repeats (premutation) may show premature ovarian failure accounting for $16 \%$ of cases of familial premature menopause in women who are normal in all other respects.

This is not a book for the family planning doctor wanting guidelines for clinical management. Rather it is a comprehensive reference and value for money (priced at £111), more for those who are curious to enquire into the scientific basis of clinical medicine.

Reviewed by David T Baird, MB BChr, DS Professor Emeritus, University Department of Obstetrics and Gynaecology and Director of Contraceptive Development Network, Edinburgh $U K$

Sex on the Rates: Memoirs of a Family Planning Doctor. Libby Wilson. Glendaruel, PK: Argyll Publishing, 2004. ISBN: 190283170 5. Pages: 224. Price: $£ 7.99$ (paperback)

I was intrigued to read the descriptions in this account of Libby Wilson's experiences in both general practice and family planning services in the sixties. Although I recognised many of the scenarios from my own experience (just a few years later than Libby), I thought as I read them will younger people believe we really managed like this? Fitting an intrauterine device (IUD) at home, while being licked by a large Alsatian dog, is a far cry from the antiseptic surroundings of most IUD fittings today. Some of the battles to provide services that she describes are continuing today - and reading historical accounts can help to avoid repeating the same mistakes.

However, I am not sure for whom the book is intended. Perhaps it is just as a record of what Libby Wilson did and achieved in a world where concealment of sexual activity and prejudice was even more common than today. These attitudes persist in some sectors of society. Those concerned with providing contraception an sexual health services sometimes need reminding why it is so difficult to set up services, find the money and resources, and prevail against people who still think that sexual activity should be punished by disease, pregnancy or shame.

The chapters on abortion and on injectable contraception illustrate the difficulties that we had in introducing both into mainstream medical practice. The risk of prosecution when offering contraception services to the under-sixteens was very real. The amount of detail included here and occasionally in other chapters makes the book more suitable for those with some knowledge of medicine, drugs and the health service. The inclusion of an index also suggests that people might want to use it as a reference.

However, the earlier chapters about Libby Wilson's childhood are suitable for anyone interested in the social history of growing up in a general practitioner's family. The account of her married life with repeated pregnancies reminds everyone of why family planning is so beneficial to modern life as a woman. I was impatient in the earlier chapters to get on - when was she going to write about 'Sex on the Rates'? Her anecdotes of the social interactions in the poorer areas of our cities resound with realism for those who work in such areas now, and she brings the book up-todate with her accounts of people using illegal drugs and suffering from AIDS. The stories about real people make the book, which is written by a remarkable and independently minded woman and doctor

Reviewed by Gill Wakley, MD, MFFP

Visiting Professor in Primary Care Development, Staffordshire University and Freelance GP Writer and Lecturer, Abergavenny, UK

\section{Visit the Faculty Website at: www.ffprhc.org.uk}

